\title{
Comparing C- and L-band SAR images for sea ice motion estimation
}

\author{
J. Lehtiranta, S. Siiriä, and J. Karvonen \\ Finnish Meteorological Institute, Marine Research Programme, Helsinki, PB 503, 00101 Finland \\ Correspondence to: J. Lehtiranta (jonni.lehtiranta@fmi.fi)
}

Received: 22 April 2014 - Published in The Cryosphere Discuss.: 26 May 2014

Revised: 31 October 2014 - Accepted: 3 November 2014 - Published: 17 February 2015

\begin{abstract}
Pairs of consecutive C-band synthetic-aperture radar (SAR) images are routinely used for sea ice motion estimation. The L-band radar has a fundamentally different character, as its longer wavelength penetrates deeper into sea ice. L-band SAR provides information on the seasonal sea ice inner structure in addition to the surface roughness that dominates C-band images. This is especially useful in the Baltic Sea, which lacks multiyear ice and icebergs, known to be confusing targets for L-band sea ice classification. In this work, L-band SAR images are investigated for sea ice motion estimation using the well-established maximal crosscorrelation (MCC) approach. This work provides the first comparison of L-band and C-band SAR images for the purpose of motion estimation. The cross-correlation calculations are hardware accelerated using new OpenCL-based source code, which is made available through the author's web site. It is found that L-band images are preferable for motion estimation over C-band images. It is also shown that motion estimation is possible between a C-band and an L-band image using the maximal cross-correlation technique.
\end{abstract}

\section{Introduction}

The Baltic Sea gets an ice cover every winter, covering $45 \%$ of its area on an average year. In the northern Bay of Bothnia, the typical duration of ice cover is from late October to late May, and the greatest level ice thickness ranges from 50 to $110 \mathrm{~cm}$. The bay has an average depth of $41 \mathrm{~m}$ and typically has large areas of landfast ice on the eastern and northeastern coasts (Myrberg et al., 2006). Observations of the Baltic sea ice are for winter navigation safety. Work has been done to calculate sea ice motion from two consecutive satellite im- ages using different optical flow estimation algorithms (e.g., Fily and Rothrock, 1987; Vesecky et al., 1988; Liu et al., 1997; Karvonen et al., 2007; Thomas et al., 2011), and this approach has provided acceptable results using the C-band synthetic aperture radar, which is regarded as a good compromise for sea ice remote sensing (Dierking and Busche, 2006). This work will compare C-band (38-75 mm wavelength) with L-band (150-300 mm wavelength) for sea ice motion estimation.

Motion estimation from consecutive satellite images has its limitations. Only an average velocity can be determined, and that only if the ice surface remains mostly unchanged. Weather conditions can change ice surface properties enough to make feature detection impossible. Generally the method only works for image pairs typically less than 3 days apart, naturally depending on the rate of the ice drift and deformation. Previous work has also concentrated on sequential images from a single instrument, which places a limitation on the availability of suitable image pairs. A satellite might fly over the area of interest only once per day or less. For longer time intervals, velocities due to short-duration events such as storms are lost.

If observations from multiple satellites are used, image pairs mere hours apart are easier to find, but the benefit comes with the added difficulty of comparing images of fundamentally different character. To improve the situation, this work will examine the idea of calculating sea ice motion using two pictures from different instruments, namely EnviSAT ASAR (56.2 $\mathrm{mm}$ wavelength), RadarSAT-2 SAR (55.5 mm wavelength) and ALOS PALSAR (236 mm wavelength). 


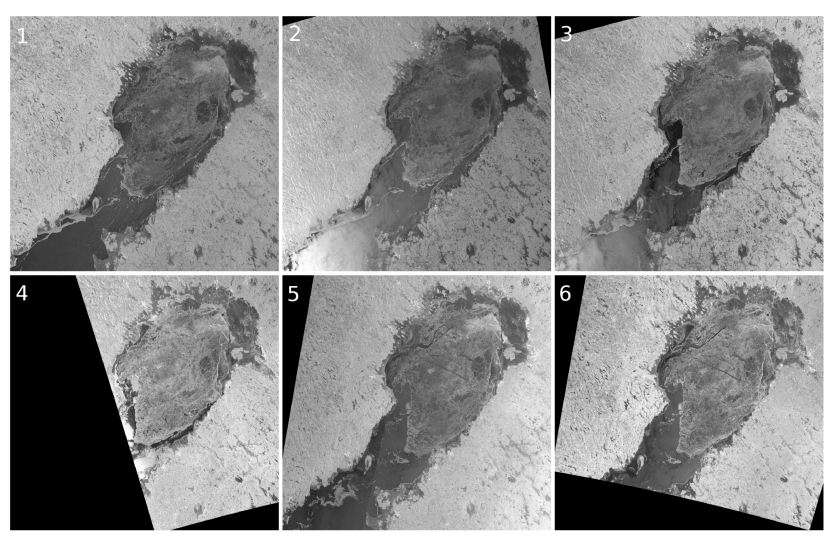

Figure 1. Satellite images used in this work, normalized for viewing. Details given in Table 1. CMDA, ESA and JAXA.

Table 1. List of satellite images used in this work.

\begin{tabular}{llllll}
\hline$\#$ & tag & satellite & time (UTC) & $t$ & band \\
\hline 1 & R1 & RadarSAT & 16 Mar 2009, 04:59 & $t_{0}$ & C \\
2 & E1 & EnviSAT & 16 Mar 2009, 19:54 & $t_{0}+14: 55$ & C \\
3 & R2 & RadarSAT & 17 Mar 2009, 16:00 & $t_{0}+35: 01$ & C \\
4 & A1 & ALOS & 17 Mar 2009, 20:12 & $t_{0}+39: 13$ & L \\
5 & E2 & EnviSAT & 18 Mar 2009, 09:04 & $t_{0}+51: 05$ & C \\
6 & A2 & ALOS & 18 Mar 2009, 09:36 & $t_{0}+51: 37$ & L \\
\hline
\end{tabular}

\section{Data and methods}

For this work, a set of synthetic-aperture radar (SAR) images from March 2009 were used (see Fig. ). C-band images were available from both EnviSAT ASAR and RadarSAT 2, while L-band images were available from ALOS PALSAR. A set of six images were chosen for the time period between 16 and 18 March. These days were chosen because there were a relatively large number of images available, including two L-band images. Additionally, two of the images were of different frequency bands and almost simultaneous, with only $32 \mathrm{~m}$ between them. This is desirable for comparing frequency bands, and a unique occurrence in the set of images that were available. The images were resampled to $100 \mathrm{~m}$ pixel size, approximately corresponding to the nominal resolution of the employed ScanSAR capturing mode.

Lots of changes including compaction and lead opening were present during this period. Landfast ice and open water areas were seen in visual inspection, as well as different types of drift ice. As the ice cover in other parts of the Baltic was sparse, only the seas north of $63^{\circ} \mathrm{N}$ latitude were considered.

\subsection{Weather and ice conditions during the experiment period}

For the Baltic Sea, the winter 2008-2009 was milder and shorter than average. Freezing commenced in the Bay of Bothnia in the second half of November, but the ice cover ex-

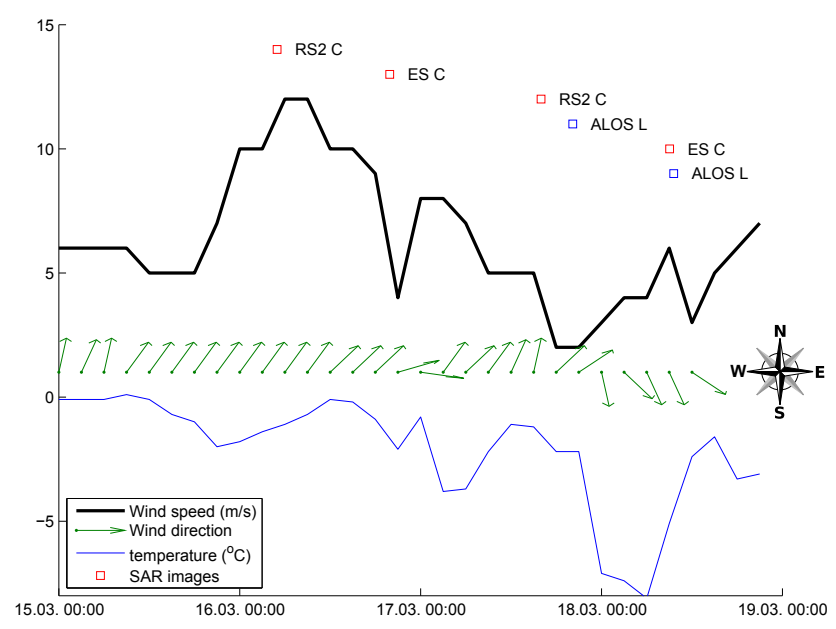

Figure 2. Wind and air temperature recorded by the Kemi 1 lighthouse weather station $\left(65.385^{\circ} \mathrm{N}, 25.096^{\circ} \mathrm{E}\right)$ during the experiment period. Timing of SAR images is also marked, red for C-band and blue for L-band images.

tended across the Bay of Bothnia only in the end of January. February was a normal winter month, and the maximum ice cover, $110000 \mathrm{~km}^{2}$, was recorded on 20 February. Much of this ice was thin, and after a cold period, warmer southwesterly winds pushed ice northwards during March. On March 16, only the Bay of Bothnia and northern Gulf of Finland had a significant ice cover (The Baltic Sea Portal, 2009).

Figure 2 summarizes the weather conditions recorded by a weather station at the Kemi 1 lighthouse (located at $65.385^{\circ} \mathrm{N}, 25.096^{\circ} \mathrm{E}$ ) during the acquisition of the satellite images. During 16 and 17 March, strong southwesterly winds were pushing the ice pack towards the north. Eventually the wind turned north. On 18 much of the ice had returned southwards and new leads had formed. The temperature remained at or below the freezing point. It is assumed that no significant melting took place during the experiment and that melting did not affect the motion estimation results. Formation of new ice, however, needs to be taken into account.

As reported in ice charts, most of the drift ice in the Bay of Bothnia is deformed, mostly by ridging but also rafting. Not much level ice remains, the well-defined areas being west of the island of Hailuoto and southwest from Tornio. There is no new ice to be found, but large sections of landfast ice lie around the coastline. Reported level ice thicknesses range from 10 to $50 \mathrm{~cm}$ in the drift ice and up to $70 \mathrm{~cm}$ in landfast ice. Six icebreakers were on duty assisting ships.

\subsection{The motion estimation approach}

For this work, a straightforward block cross-correlation program was written in the general purpose $\mathrm{C}++$ programming language. The code works directly in the spatial domain, to allow normalized cross-correlation, more flexibility in finetuning the computational parameters (Emery et al., 1991) and 


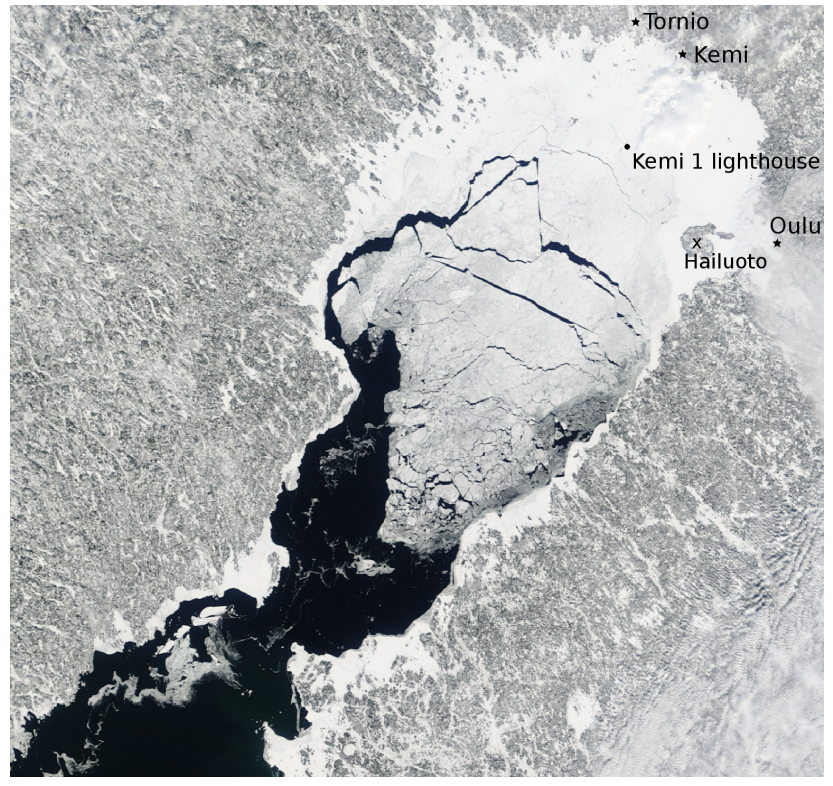

Figure 3. True colour satellite image of the Bay of Bothnia, 18 March 2009, 10:05 UTC. Image captured by the MODIS instrument on board the Terra satellite, courtesy of NASA.

easy parallelization. Critical parts of the algorithm were programmed in OpenCL $\mathrm{C}$, which is a portable language for writing code that can be run in a parallel fashion on a variety of devices (Stone et al., 2010). The cross-correlation code was run on a Graphics Processing Unit (GPU) produced by NVIDIA. This approach cut down the calculation time significantly. The OpenCL cross-correlation program can process one pair of images in roughly $20 \mathrm{~s}$, as opposed to 20 min for a single-core program running on the CPU. This source code is available through the author's website at http://jonni.lehtiranta.net/.

The motion vectors were calculated using a multiresolution approach. This is usually done to limit the area that has to be processed, but because of the GPU approach, only $48 \mathrm{kB}$ of fast local memory was available. The size of the search domain was limited to $96 \times 96$ pixels. First, motion vectors were calculated in a coarse resolution $(1 / 8$ of the original or $800 \mathrm{mpixel}^{-1}$, which allows almost $40 \mathrm{~km}$ displacements), and median-filtered result vectors were used as initial guesses for the high-resolution matching step. Finally, the high-resolution result was median-filtered to remove problematic values. For this work, the median filtering radius was chosen to be 3 (as in Karvonen et al., 2007).

For the image windows that were cross-correlated with the search domain, a size of $16 \times 16$ pixels was chosen. There is a tradeoff involved in choosing this window size, as it has to be large enough to contain a discernible pattern and at the same time small enough to retain its structure in the time interval separating the pair of images. The chosen size is at the small end of practical options. It was chosen to minimize errors due to deformations, and to concentrate on errors due to lack of discernible patterns within these windows. This way the error fractions are maximally useful for comparing C-band images to L-band images.

The method consists of the following steps:

1. re-projecting and cropping satellite images using the GDAL toolset,

2. loading the GeoTIFF images, translating 16-bit greyscale values to floating-point numbers,

3. generating a resolution pyramid for both images, using a 2-D low-pass filter and decimating for every level,

4. running normalized cross-correlation for coarseresolution image windows,

5. median-filtering the coarse result to produce the average motion field and first guess for next step,

6. running normalized cross-correlation for the finestresolution image windows,

7. saving this result and a median-filtered version (radius 3) of it in an ASCII text file.

The results were analysed and plotted using the Matlab and Octave programs.

\subsection{Performance metrics for motion estimation}

For this study, no ground truth data was available for comparison. It was necessary to define some performance metric that could be calculated from the results alone. In this work, the cross-correlation method was not tuned for the image types, and especially between C- and L-band images, low crosscorrelation coefficients were expected. Instead of the crosscorrelation coefficient itself, we consider the ratio of the two highest peaks. While a high peak-to-peak ratio is not conclusive evidence of correctness, it is assumed to be a necessary requirement. A motion vector is rejected if the margin between two highest cross-correlation peaks is less than $15 \%$, and otherwise accepted in a "peak margin" sense.

Additionally, each motion result is evaluated against the expectation of uniformity, flagging as errors all vectors that differ significantly from the median-filtered vector field. It is assumed that the median filtering succeeds at removing spurious values and retains real stepwise changes in the ice motion field (Astola et al., 1990), so that the median-filtered motion field represents the real average motion. Even when this is not the case, unrealistic vectors will not match it so these cases cannot produce false successes. A motion vector is rejected if it differs from the median of its neighbourhood by more than $500 \mathrm{~m}$. Otherwise it is considered acceptable in a "regularity" sense.

Both criteria are arbitrary. However, they appear to be sensible choices for this study. 


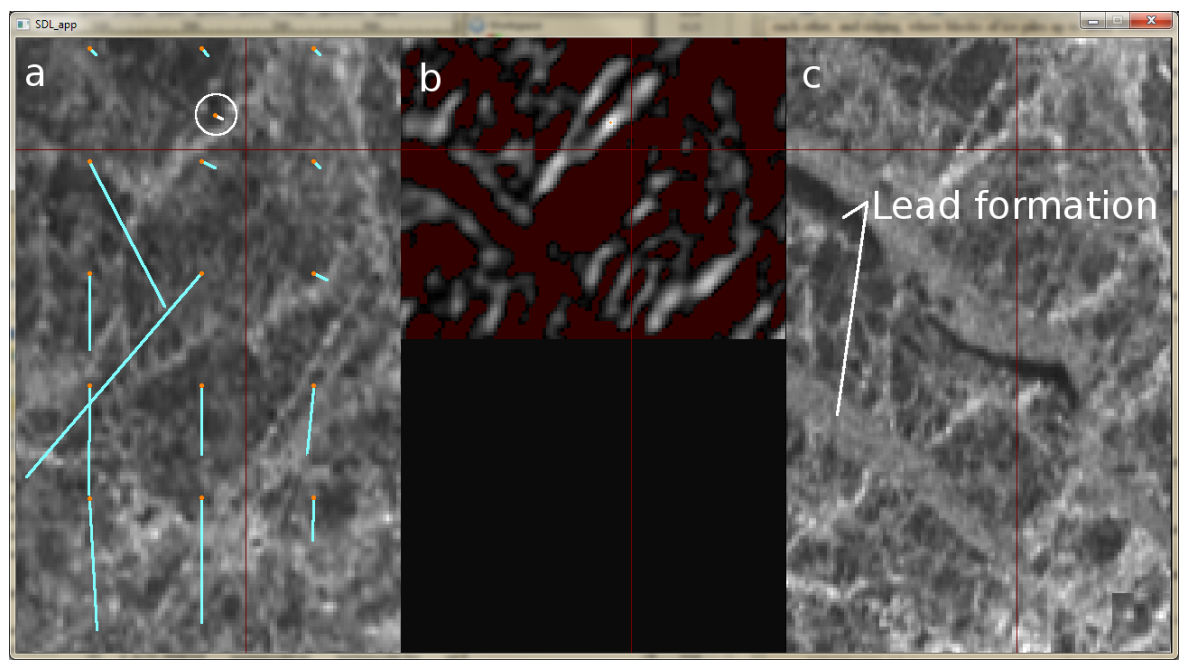

Figure 4. Screenshot of the motion estimation program written for this work. (a) Zoom-in of the first image with some detected motion vectors. (b) The cross-correlation result for the circled vector. White represents maximum cross-correlation, black represents zero correlation and the area left outside of the calculation. Red represents negative cross-correlation. (c) Aligned zoom-in of the second image of the pair. Notice the newly formed NW-SE aligned leads. The thin red lines are rulers that highlight the mouse cursor's location.

\subsection{Satellite image processing}

Algorithms used for operational satellite image analysis are often tuned to the specific instruments. As the objective of this study is to compare different instruments, no instrumentspecific tuning was done. The images still need georectification, and typically a landmask is used.

For this work, SAR images are rectified to the Mercator projection with a reference latitude of $61^{\circ} 40^{\prime}$. This projection was chosen as it matches the one used in both the nautical charts for this area and previous ice motion estimation work for the Baltic Sea (Karvonen, 2012). There still remains a slight error after this projection step. It could be corrected by matching static features between the images.

An incidence angle correction was not performed. It was deemed unnecessary, as the method calculates normalized cross-correlations for small image windows. No speckle filtering was applied.

\subsection{Masking land points}

For sea ice motion estimation in the narrow basins of the Baltic Sea, land points are sometimes masked out before analysis (Karvonen, 2012). In this work, motion detection was performed using unmasked images. Result vectors for land and sea areas were then analysed separately. As a drawback, image windows that include the coastline generate two valid cross-correlation peaks. Land points and shallow areas were distinguished by topographical data produced by the Leibniz Institute for Baltic Sea Research (Seifert et al., 2001).

The satellite images were found to suffer from a spatially varying registration error. This was corrected using the finest- resolution motion estimates for land points. These were interpolated in order to generate a seamless estimate for the image registration error. This registration error field was finally substracted from the motion results recorded for the drift ice.

\section{Visual comparison between L- and C-band images}

The PALSAR L-band images have been compared to RADARSAT-1 SAR by the Canadian Ice Service. They report that the L-band images contain a far superior amount of ridge information compared to C-band. Large ridges are clearly defined, and detail remains well into the spring melt season. It is also reported that PALSAR allows clearer delineation between ice floes. PALSAR also allows thin ice to be easily distinguished from thick ice, while C-band images could confuse rough thin ice with thicker ice types (Arkett et al., 2008).

As images 5 and 6 (see Table 1 and Fig. ) are separated by only $32 \mathrm{~min}$, they are assumed to represent the same ice situation in C- and L-bands. No ice-related change can be distinguished visually, so all differences are taken to result from differences between the imaging instruments. As a general difference, the L-band image (f) has more contrast within the sea area. The coastline is also more easy to distinguish, while in the C-band image, the coastline disappears in some, especially northern, locations. Below, specific differences in these two images are evaluated in detail.

To summarize, ice types in the drift ice region appear similarly in images of both frequency bands. Sometimes the Cband image is better at distinguishing the edge of an ice floe, and sometimes the L-band shows features not visible in the C-band image (see east edge of Fig. 9), but for most features, 


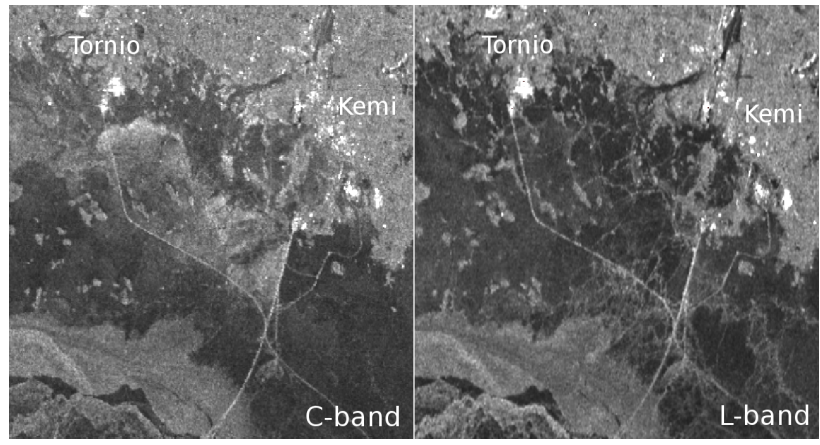

Figure 5. Detail of landfast ice in northern Bay of Bothnia on 18 March 2009. White tracks are shipping lanes to Tornio and Kemi, which appear very bright in SAR images.

the L-band image simply seems to provide stronger contrast. On the other hand, many features in landfast ice appear differently in C- and L-band images. Perhaps a long, relatively peaceful evolution of an ice surface produces surface roughness in length scales comparable to the radar wavelengths.

\subsection{Landfast ice}

Landfast ice is immobile and non-dynamic by definition. It is assumed that no recent deformation took place in the landfast zone. Discernible features are assumed to be either old deformations or weather-related. As can be seen in Fig. 5, the archipelago looks more homogenous and dark in the L-band image. Conversely, the C-band image shows a large hazy feature, conspicuously framed by the shipping lanes.

The linear or web-like features visible in the L-band image but missing from the $\mathrm{C}$-band image are probably due to the greater volume scattering in L-band. The surface scattering is weaker and less extended, perhaps due to snowfall or meltfreeze events.

Features missing from the L-band image but visible on the C-band image, on the other hand, are probably caused by surface roughness smaller than the L-band wavelength $(23.6 \mathrm{~cm})$. The shipping lanes that constrict the bright haze in the $\mathrm{C}$-band image, provide a hint of its formation. This was possibly mobile broken slush, which froze to form a rough surface on the northern side of the shipping lanes.

Near the southwest corner, there is a brighter gray band without clear features. This is the shear zone at the landfast ice boundary, experiencing deformation by external forces but still attached to the landfast ice, islands, or the shallow sea floor. The dark feature under it is open water or thin ice in a lead, and we also see some drift ice in the corner of the image. These features look similar in both images.

In Fig. 6, the L-band image has ill-defined bright features in the landfast ice zone while the C-band shows little scattering. To know the evolutionary history of these features, one would need to track their formation from the beginning of the freezing period. Here, too, early-season deformations could

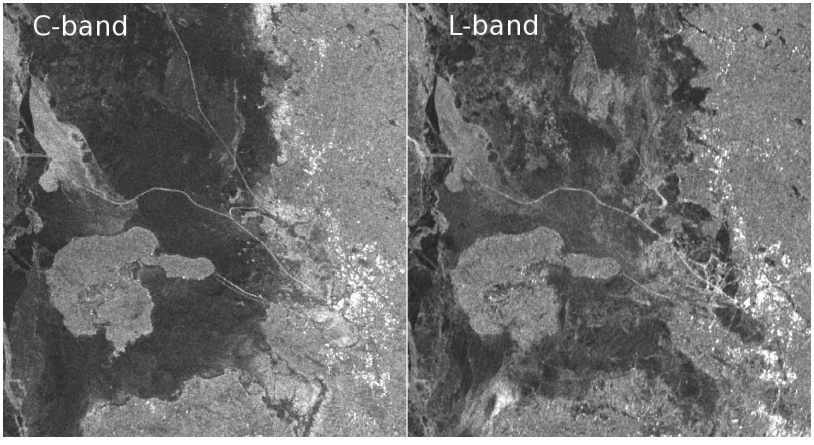

Figure 6. Detail of landfast ice in northern Bay of Bothnia around Hailuoto, offshore from Oulu, on 18 March 2009.

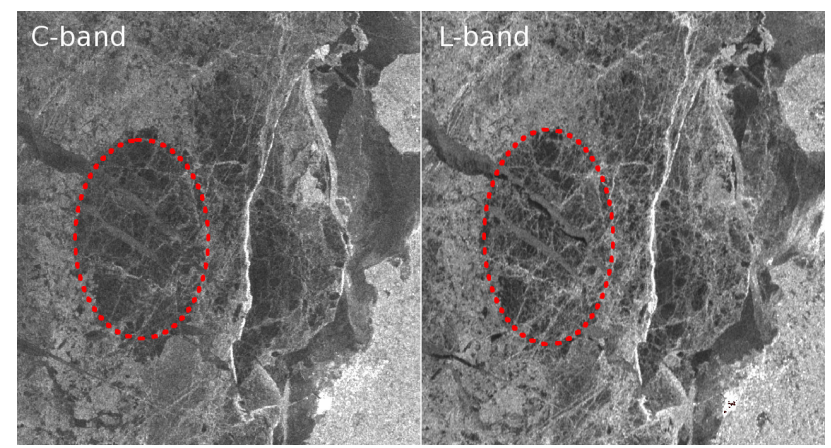

Figure 7. Elliptic dark area classified as level ice near Raahe on 18 March 2009.

be masked by smoothing surface processes. The bright feature north of Hailuoto island, which appears similar in both images, is probably a field of broken ice, often called a rubble field, analogous to a very wide pressure ridge.

Comparing these images, it can be concluded that landfast ice can be a tricky substance for matching windows of SAR images of different bands. Some features will appear similar but at different intensities, and some areas will look completely different.

\subsection{Level ice}

Some ice classified as level ice can be seen in the southwest corner of Fig. 6, southwest from Tornio in Fig. , and in the dark ovals in Fig. 7. These areas show up as relatively dark areas, presumably because of relatively low specular reflection, in SAR images of both wavelengths. In general, Cband shows these features darker than L-band, as L-band will cause more scattering from beneath the level surface (Dierking and Busche, 2006). In some areas, level ice is relatively featureless and in others rather detailed. Some of the areas look identical in C- and L-bands, others show more contrast in L-band. However, based on visual inspection, correlating image windows in level ice seems feasible. This analysis is limited by the small amount of level ice. 

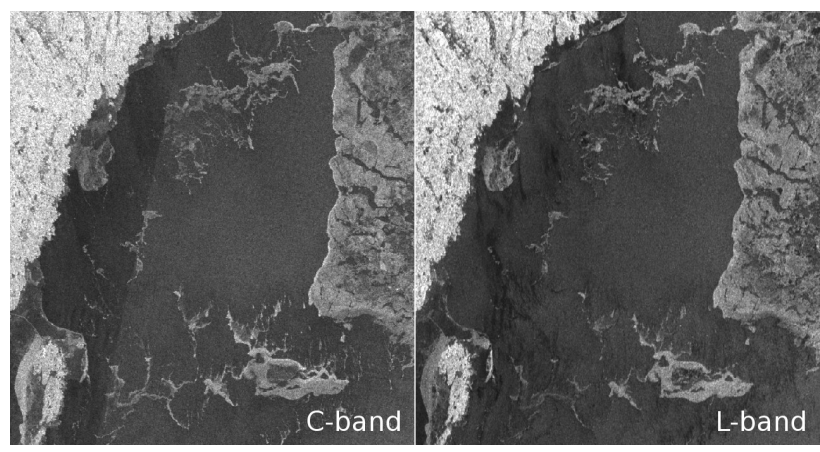

Figure 8. Open ice between the Swedish coast and the compact ice pack in North Kvarken on 18 March 2009.

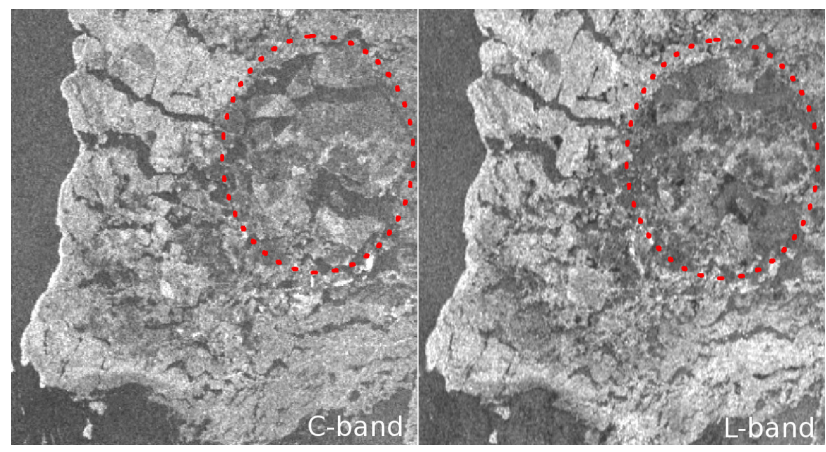

Figure 9. Southern tip of the compact drift ice on the Bay of Bothnia on the 18 March 2009. Encircled the area of faint, barely distinguishable ice floes.

\subsection{Open ice}

Sea areas with less than $60 \%$ ice cover are classified as open ice. In open ice, separate ice floes drift freely among waves. Using both frequency bands, ice forms similar gray curls, visible in Fig. 8, that should allow motion detection using cross-correlation to work well. Most notable visible differences are dark lines in the open water in the L-band image, and slightly better contrast in the C-band image. However, these formations appear fragile and susceptible to changes, which makes tracking them rather demanding.

\subsection{Compact drift ice}

Drift ice, classified in Finnish ice maps as consolidated, compact or very close ice, often covers the central Bay of Bothnia during winters. It is a mobile continuum, it deforms readily and it transmits compressive forces over large distances.

In Fig. 9, separate but closely packed floes of compact drift ice can be seen, sometimes separated by leads or other open water features. Many distinct ice floes are recognizable in both images, but the fainter floes near the eastern edge are not visible in the L-band image despite standing out very clearly in the true-colour Fig. 3. The L-band image seems less able

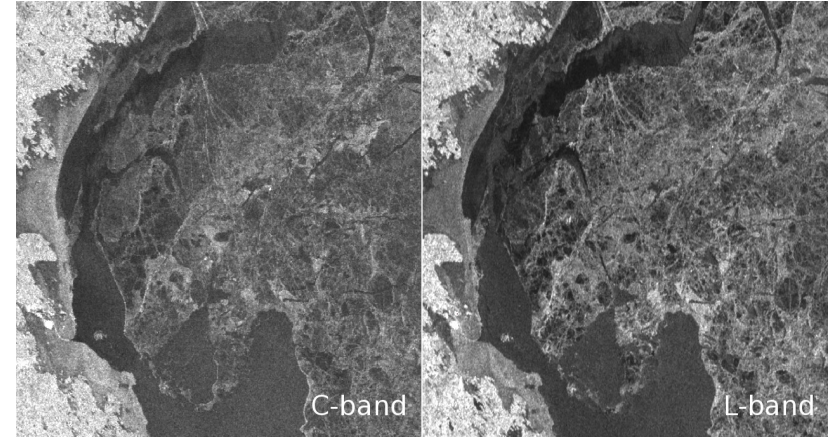

Figure 10. Drift ice on the western Bay of Bothnia, 18 March 2009.

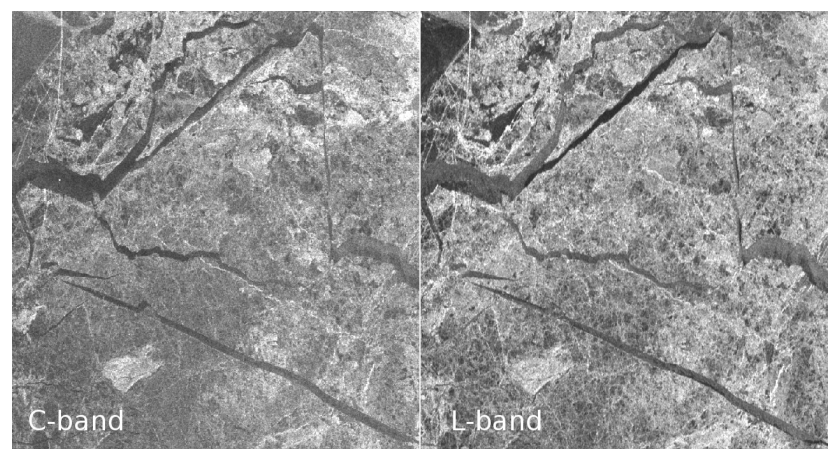

Figure 11. Leads in drift ice, Bay of Bothnia, 18 March 2009.

to distinguish the edge between a lead and a smooth ice floe. Occasionally there is texture not present in the C-band image, such as the bright features in the southeast corner. However, the edge of open water is well visible and similar in both frequency bands, and most ice floes are similar enough for motion estimation.

In Fig. 10, a compact and mostly continuous ice pack is seen in both C- and L-band. Both images reveal the same features, though L-band in better contrast.

It is evident from Figs. 10 and 11 that sometimes leads appear very dark in L-band images. In general however, leads are visible in both kinds of images, and should pose no special problem for motion estimation in a mixed-frequency image pair.

\section{Results and discussion}

\subsection{Motion estimates}

To summarize, the motion estimates calculated for image pairs covering the same time interval are similar in all cases. For a $\mathrm{C}-\mathrm{C}$ or $\mathrm{L}-\mathrm{L}$ band image pair, the matching is better and motion results may be found for a larger area than in a mixed pair. Based on the metrics defined in Sect. 2.3, an L-L image pair is superior for motion estimates compared to $\mathrm{C}-\mathrm{C}$ pairs, 

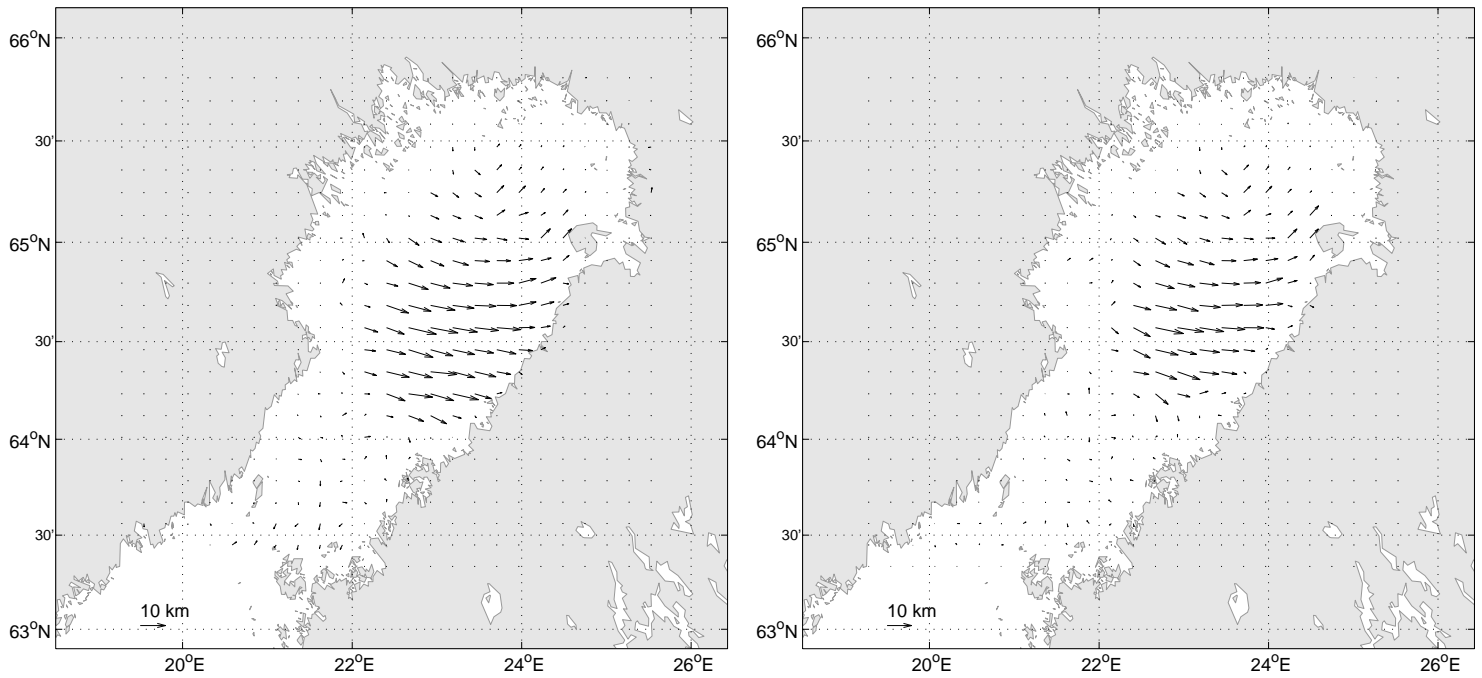

Figure 12. (a) Motion vectors from combining images 1 and 6, of C- and L-band, respectively. (b) Motion vectors from combining images 1 and 5, both C-band.
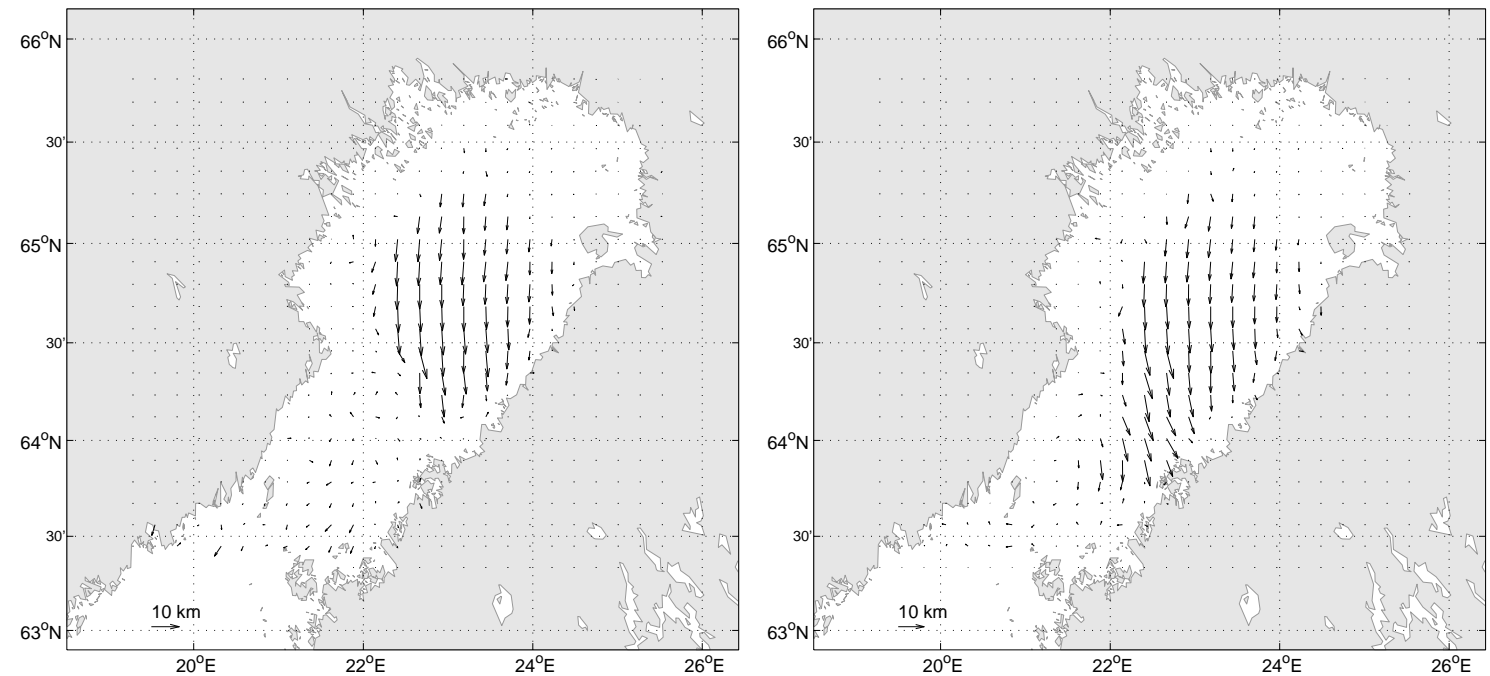

Figure 13. (a) Motion vectors from combining images 2 and 6, of C- and L-band, respectively. (b) Motion vectors from combining images 2 and 5, both C-band.

while mixed pairs are still feasible despite them presenting the most problematic case.

The average motion for the whole experiment period is shown in Fig. 12. Both a $\mathrm{C}-\mathrm{C}$ pair and a mixed $\mathrm{L}-\mathrm{C}$ pair produce an acceptable result for most of the drift ice. The motion fields are almost identical, and the average eastward motion is well supported by the southwesterly winds that turned north towards the end of the period. It is notable though, that neither image pair produces motion for the southern tip of the drift ice area. This is probably because the ice edge changed shape completely, and the numerous ice floes were too small to be distinguished. These two parallel estimates correspond to the R1-A2 and R1-E2 rows in Table 2. Of the motion vectors in the R1-A2 image pair, $17.6 \%$ had an acceptable cross-correlation peak margin, and $14.0 \%$ of the vectors were close to the local median. For the concurrent image pair R1-E2, both C-band, an additional $2 \%$ of the motion vectors passed both criteria.

In Fig. 13, we see an average southward movement for the latter $36 \mathrm{~h}$ of the experiment. This is in line with the prevailing winds as well, as the northward transport of ice had stopped before the winds turned north. This time, for the C-band pair, the southern ice edge is also successful but Fig. 13a shows no motion where Fig. 13b finds realistic vectors. These two parallel estimates correspond to the E1-A2 and E1-E2 rows in Table 2. Again, the $\mathrm{C}$-band pair produces 

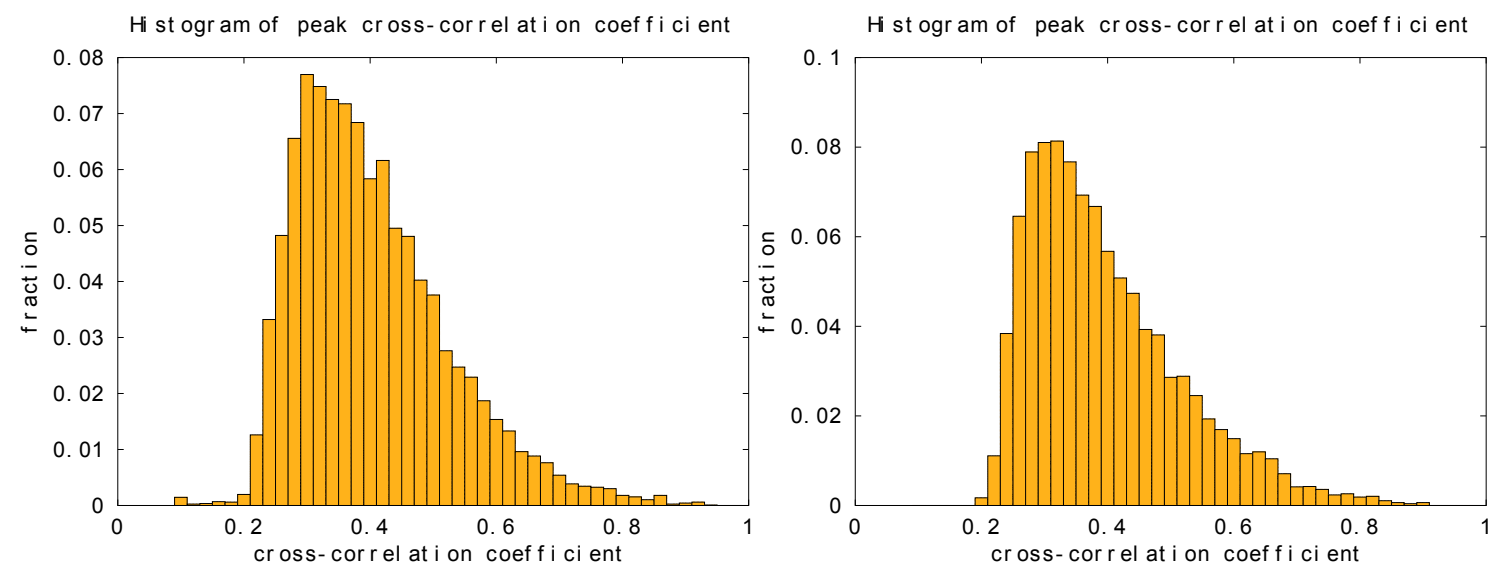

Figure 14. Maximum cross-correlation for matched windows in the R2-A2 image pair (C-L, left) and the R2-E2 image pair (C-C, right)
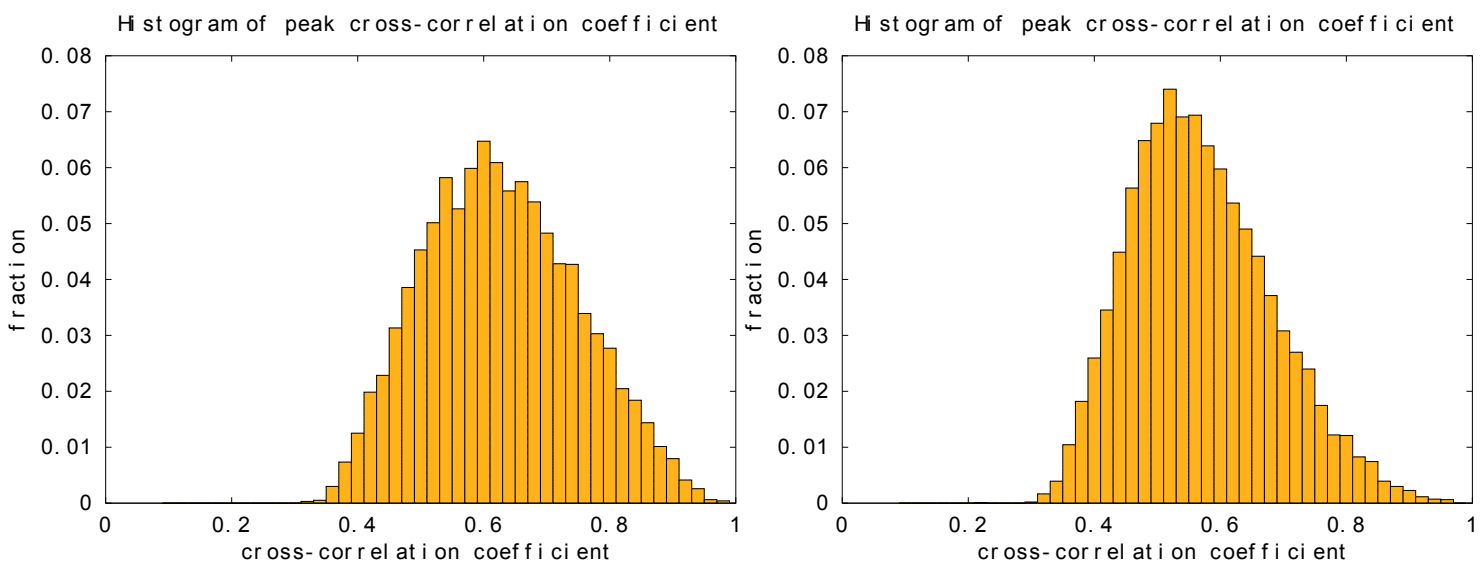

Figure 15. Maximum cross-correlation coefficient histogram for the A1-A2 image pair (L-L), left, and the A1-E2 image pair (L-C), right.

more acceptable vectors, some of which must be located in the southern ice edge, less deformed during the shorter time span covered by these image pairs.

The four latter motion estimates, represented on the two bottom rows of Table 2.3, appear very much like Fig. 13b. This is because each of these image pairs cover the whole period of northerly winds.

Comparing the performance of parallel image pairs, some observations were made. As expected, the motion estimation algorithm works better for shorter timescales, as less deformation has had time to occur. For all image pairs, large-scale motion estimation was successful. All motion estimates contained a large number of spurious vectors too, but a radius 3 median filtering was found to produce a realistic and smooth motion field. Due to the median filtering, the algorithm works even if only $10-20 \%$ of motion vectors are correct. This success rate is thus found sufficient for detecting the large-scale motion. However, as evident in Fig. 13, a mixed image pair can fail in details in some sub-regions.

Same-band image pairs $(\mathrm{C}-\mathrm{C}, \mathrm{L}-\mathrm{L})$ are found better than mixed-band $(\mathrm{C}-\mathrm{L})$ pairs. Further, the L-band is found more suitable for motion estimation in this data set than C-band. Unfortunately, it seems that a large peak margin in crosscorrelation is not sufficient as an indicator of correctness. Many motion vectors were found to be nonsensical even when they were produced by a unique cross-correlation peak. This can happen, for example, when the ice surface pattern is lost between images. Upon closer investigation, it was found that a motion estimate using the highest peak is often correct even if the second-highest peak is just barely lower.

\subsection{Statistical performance of image pairs}

Overall, both C- and L-band image pairs and mixed image pairs show similar statistical properties in the motion results.

For most image windows, the highest found normalized cross-correlation coefficient was between 0.2 and 0.6 . The best matches had a cross-correlation coefficient up to 0.95 . As can be seen in Fig. 14, for C-band pairs the worst match is around 0.2. This is closer to 0.4 in the L-band pair of Fig. 15, which has overall higher correlation coefficients. 

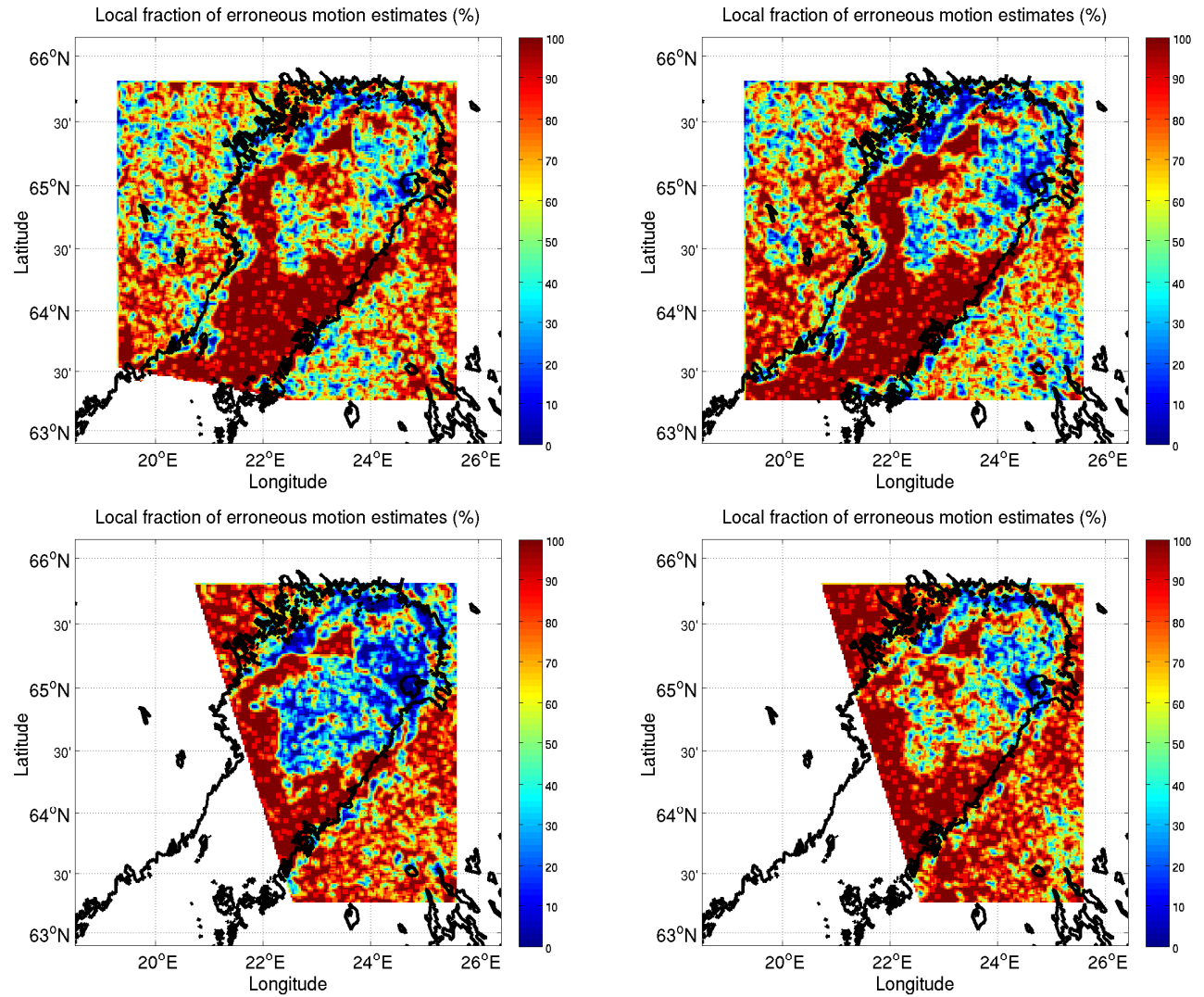

Figure 16. Geographical distributions of errors, (a) pair R2-A2 (C-L), (b) R2-E2 (C-C), (c) A1-A2 (L-L) and (d) A1-E2 (L-C)

Table 2. Performance values for parallel image pairs, as the percentage of motion vectors that are accepted based on the peak margin -criterion (pm-good) and regularity-criterion (reg-good), both defined in Sect. 2.3.

\begin{tabular}{lcc}
\hline Image pair & pm-good & reg-good \\
\hline R1-A2 (C-L) & $17.6 \%$ & $14.0 \%$ \\
E1-A2 (C-L) & $20.1 \%$ & $14.2 \%$ \\
R2-A2 (C-L) & $24.7 \%$ & $15.8 \%$ \\
A1-A2 (L-L) & $45.6 \%$ & $28.4 \%$ \\
\hline R1-E2 (C-C) & $19.6 \%$ & $16.2 \%$ \\
E1-E2 (C-C) & $22.7 \%$ & $16.7 \%$ \\
R2-E2 (C-C) & $27.9 \%$ & $18.6 \%$ \\
A1-E2 (L-C) & $30.7 \%$ & $18.7 \%$ \\
\hline
\end{tabular}

The ice conditions and their change are the most important factors of success. This is evident from Fig. 15b. The A1-E2 image pair boasts large cross-correlation coefficients despite mixing two different wavelengths.

The histograms for motion estimation error magnitude, as estimated by the difference in metres between each motion vector and the local median, are all rather similar. The histograms of error show a strong peak for no or very small error and a distribution characteristic of this problem. This distribution roughly corresponds to the idealized theoretical distribution of the distance of a random point. This distribution arises from the fact that the search window is square and it allows at most 40 pixels of movement in each dimension. It is concluded that there are no systematic errors in the motion estimation algorithm.

Considering the margin between the two highest correlation peaks, it was found that a $\mathrm{C}-\mathrm{C}$ pair is better than a mixed $\mathrm{C}-\mathrm{L}$ pair at finding unique peaks. The difference is small though, and very often the highest cross-correlation peak stands only slightly above the second contender. This was expected, as the maximal cross-correlation (MCC) method is known to often produce multiple cross-correlation peaks for noisy signals. To improve performance, the algorithm should consider multiple cross-correlation peaks, not just the highest one.

\subsection{Geographical distribution of errors}

The geographical distribution of errors was calculated for the test cases with smallest time difference in order to evaluate problems stemming from local effects and not changes that occur over longer time intervals. Figures $16 \mathrm{a}$ and $16 \mathrm{~b}$ correspond to the same time interval and show that a $\mathrm{C}-\mathrm{C}$ pair is stronger than a $\mathrm{C}-\mathrm{L}$ pair in all localities, but the mixed-band 
pair also succeeds to some extent everywhere the $\mathrm{C}-\mathrm{C}$ pair does. Figure $16 \mathrm{c}$ and $\mathrm{d}$ correspond to another time interval and shows that an $\mathrm{L}-\mathrm{L}$ pair is much better than a mixed pair, again without any clear difference in the areas of successful motion estimation.

To summarize, all image combinations have trouble with the northwesterly lead opening near the northeast edge of landfast ice, and all combinations behave better in the central ice pack. It is clear that a single-frequency pair is desirable, but also that for most regions, a mixed-frequency pair performs reasonably well. No image pair finds more than an occasional good motion vector in open ice of less than $30 \%$ coverage. It seems that the C-band is better than L-band for matching image patterns on land. While this is of no concern for perfectly georeferenced images, this might mean that georectifying $\mathrm{L}-\mathrm{L}$ image pairs might be more problematic.

\section{Conclusions}

We show that it is possible to calculate sea ice motion using an L-band SAR image together with a C-band image. The program written for this purpose works and produces convincing results, so the chosen algorithm of maximal crosscorrelation suits this purpose.

L-band images are fundamentally different than C-band images as the ratio of surface and volume scattering is different and some C-band scatterers are invisible to L-band radar. This difference manifests itself primarily in landfast ice, possibly because long periods of thermodynamical changes create different surface features near the length scales of the employed wavelengths. Fortunately, the motion estimation largely succeeds for landfast ice, and most features in drift ice appear much easier targets for motion detection.

The different frequency bands complement each other when plentiful data is available, but they are somewhat poorer for backup purposes as each band has distinct strengths and weaknesses. On C-band, ice floe edges appear in a more reliable manner, while the L-band distinguishes the coastline better and generally shows more features and better contrast.

For motion estimation, a pair of two L-band SAR images is found to be desirable among the compared options. A pair of two C-band images also performs well, and a mixed pair performs adequately. The introduction of L-band SAR instruments can thus present both more reliable motion estimates by using $\mathrm{L}-\mathrm{L}$ pairs and better time resolution, albeit at a cost of increased uncertainty, by using mixed $\mathrm{L}-\mathrm{C}$ pairs.

This work provides a new tool for motion estimation. It also provides insights into the usage of L-band SAR images, both alone and in combination with C-band images. Thus, it is good preparation for the future launch of the ALOS2 satellite and for the handling of its L-band images, and utilizing the GPGPU computational framework was both a strength in this work and a valuable lesson for the future.
Acknowledgements. This study was supported by the Finnish Meteorological Institute and the Polar View project. The authors wish to thank Eero Rinne, Lars Kaleschke and Lang Wenhui for their detailed and insightful comments on the original manuscript.

Edited by: L. Kaleschke

\section{References}

Arkett, M., Flett, D., De Abreu, R., Clemente-Colon, P., Woods, J., and Melchior, B.: Evaluating ALOS-PALSAR for Ice Monitoring-What Can L-band do for the North American Ice Service?, in: Geoscience and Remote Sensing Symposium, IGARSS 2008, IEEE International, 5, V-188, 2008.

Astola, J., Haavisto, P., and Neuvo, Y.: Vector median filters, P. IEEE, 78, 678-689, 1990.

The Baltic Sea Portal: Ice winter 2008-2009, available at: http: //www.itameriportaali.fi/en/tietoa/jaa/jaatalvi/en_GB/2009/ (last access: 18 December 2014), 2009.

Dierking, W. and Busche, T.: Sea Ice Monitoring by L-Band SAR: An Assessment Based on Literature and Comparisons of JERS1 and ERS-1 Imagery, IEEE T. Geosci. Remote, 44, 957-970, 2006.

Emery, W., Fowler, C., Hawkins, J., and Preller, R.: Fram Strait satellite image-derived ice motions, J. Geophys. Res., 96, 47514768, 1991.

Fily, M. and Rothrock, D.: Sea ice tracking by nested correlations, IEEE T. Geosci. Remote, GE-25, 570-580, 1987.

Karvonen, J.: Operational SAR-based sea ice drift monitoring over the Baltic Sea, Ocean Sci., 8, 473-483, doi:10.5194/os-8-4732012, 2012.

Karvonen, J., Similä, M., and Lehtiranta, J.: Sar-based estimation of the baltic sea ice motion, in: Geoscience and Remote Sensing Symposium, IGARSS 2007, IEEE International, 2605-2608, 2007.

Liu, A. K., Martin, S., and Kwok, R.: Tracking of Ice Edges and Ice Floes by Wavelet Analysis of SAR Images, J. Atmos. Ocean. Tech., 14, 1187-1198, 1997.

Myrberg, K., Leppäranta, M., and Kuosa, H.: Itämeren fysiikka, tila ja tulevaisuus, Palmenia, Helsinki University Press, 2006.

Seifert, T., Tauber, F., and Kayser, B.: A high resolution spherical grid topography of the Baltic Sea, 2nd Edn., Baltic Sea Science Congress, Stockholm, 25-29 November 2001, Poster\# 147, available at: www.io-warnemuende.de/iowtopo (last access: 18 December 2014), 2001.

Stone, J. E., Gohara, D., and Shi, G.: OpenCL: A parallel programming standard for heterogeneous computing systems, Comput. Sci. Eng., 12, 66-72, 2010.

Thomas, M., Kambhamettu, C., and Geiger, C.: Motion Tracking of Discontinuous Sea Ice, IEEE T. Geosci. Remote, 49, 5064-5079, 2011.

Vesecky, J. F., Samadani, R., Smith, M. P., Daida, J. M., and Bracewell, R. N.: Observation of sea-ice dynamics using synthetic aperture radar images: Automated analysis, IEEE T. Geosci. Remote, 26, 38-48, 1988. 